\title{
REGRESSÃO DE LINFOMA GÁSTRICO MALT EM TRANSPLANTE RENAL APÓS CONVERSÃO DA IMUNOSUPPRESSÃO PARA SIROLIMO
}

\author{
Malt gastric lymphoma regression in a renal transplant after conversion of \\ immunosuppression to sirolimus
} Euler Pace Lasmar, Luiz Gonzaga Vaz Coelho, Marcus Faria Lasmar, Leonardo Faria Lasmar,
Adalberto Fernandes Nogueira, Luiz Flávio Couto Giordano, Heloisa Reniers Vianna.

\begin{abstract}
RESUMO
O linfoma não-Hodgkin de células B é a disfunção linfo-proliferativa mais comum após transplante de órgão sólido e seu tratamento não é bem definido. Reportamos um caso de linfoma gástrico MALT com remissão rápida, persistente e completa após conversão da imunossupressão de ciclosporina (CsA) para sirolimo (SRL). Uma mulher de 42 anos submetida a transplante renal em 1992 evoluiu sem maiores anormalidades até 2006, quando uma gastroscopia executada para investigar sintomas dispépticos mostrou uma associação de linfoma gástrico MALT (com componentes de baixo e alto graus) com uma infecção por Helicobacter pylori(H.pylori). Foram realizadas duas intervenções terapêuticas com intervalo de uma semana: tratamento da infecção por $H$. pylori (associação de omeprazol, amoxicilina e claritromicina por 14 dias) e modificação da imunossupressão, substituindo a CsA e azatioprina (AZA) por SRL. Ecoendoscopia de controle executada um mês depois mostrou persistência da infecção por $H$. pylori e ausência do tumor gástrico. Novas ecoendoscopias executadas aos dois, sete meses e um ano após a terapia confirmaram a ausência da neoplasia e erradicação do H. pylori. Atualmente, a paciente não apresenta queixas, com valores de creatinina de $1,8 \mathrm{mg} / \mathrm{dl}$, fazendo uso de SRL e ibersatan. A SRL tem sido extensivamente estudada como droga anticancerígena, atuando como inibidor do mTOR (mammalian target of rapamycin). Dados acumulados na literatura suportam o papel do mTOR na linfomagênese. Pelo que sabemos, este é o primeiro relato de linfoma gástrico MALT em paciente submetido a transplante renal com remissão completa após alteração do esquema de imunossupressão com a introdução de SRL.
\end{abstract}

Descritores: transplante renal, linfoma Malt, imunossupressão

\section{Instituição:}

Unidade de Transplante Renal do Hospital Universitário São José / Hospital Mater Dei Belo Horizonte / MG - Brasil

\section{Correspondência:}

Euler Pace Lasmar

Rua Newtow, 89 - Belo Horizonte / MG - CEP: 30360-200 - Brasil

Tel.: (31) 32978848

E-mail: lasmar@superig.com.br

Recebido em: 26.08 .2008

\section{INTRODUÇÃO}

As doenças linfoproliferativas no pós-transplante ocorrem em 1 a $20 \%$ de receptores de transplante de órgãos sólidos, a maioria delas determinadas pelo linfoma não-Hodgkin de células B. ${ }^{1} \mathrm{~A}$ ocorrência em receptores renais está associada a um maior risco de morte e à diminuição da taxa de sobrevida de $80 \%$ para $65 \% \mathrm{em}$ cinco anos. ${ }^{2} \mathrm{O}$ tratamento não está bem definido e determina um risco significante de rejeição ou perda da função renal. Tem sido demonstrado que o sirolimo (SRL), uma droga imunossupressora inibidora do mTOR (mammalian target of rapamycin), exibe propriedades anti-tumorais in vitro e in vivo. Pelo que sabemos, este é o primeiro relato de regressão de linfoma gástrico MALT (LGM) em paciente transplantado renal após conversão de ciclosporina (CsA ) para SRL.

\section{RELATO DE CASO}

Uma mulher de 42 anos, branca, submetida a transplante renal em 03/06/1992 com doadora irmã (três mismatches) evoluiu sem anormalidades durante o período imediato pós-operatório, recebendo tratamento imunossupressor com CsA, azatioprina (AZA) e prednisona.(PRED). Apresentou um episódio de rejeição 
aguda em 1994, controlado com pulsoterapia (metilprednisolona, 250 mg x 3 dias). Em janeiro de 2001 apresentou quadro de papiloma vírus vaginal, quando a dose de AZA foi reduzida (100 - $75 \mathrm{mg})$. A paciente foi submetida à endoscopia digestiva alta em 07/06/2006 devido a sintomas dispépticos, tendo sido feito diagnóstico histológico e imunohistológico de linfoma gástrico MALT (com componentes de baixo e alto graus) associado à presença de H.pylori (Figuras 1 e 2). O tratamento para erradicação do H.pylori foi iniciado em 13/06/2006, com o uso de omeprazol, amoxicilina e claritromicina por 14 dias. Em 20/06/06, foi alterada a imunossupressão, com a substituição do CSA e AZA por SRL dose de $4 \mathrm{mg} /$ dia. Naquela epoca, o nível de creatinina era de 2,1 $\mathrm{mg} / \mathrm{d}$ e a sorologia para Epstein Barr-vírus (EBV) para IgM e IgG foi positiva. As tomografias computadorizadas (TC) do cérebro, abdômen e tórax foram normais. A endoscopia digestiva superior de controle realizada em 12/7/2006 mostrou apenas gastrite crônica ativa com atrofia e metaplasia intestinal, ainda associada à presença de H.pylori. Não se encontrou mais neoplasia (Figura 3). A ecoendoscopia apresentou apenas espessura localizada da parede gástrica envolvendo predominantemente as camadas de mucosa e submucosa, além de pequenos e inespecíficos nódulos linfáticos peri gástricos. Novas endoscopias foram executadas em 21/08/2006, 08/01/2007e 25/09/2008 evidenciando ausência de neoplasia e H.pylori. Atualmente, a paciente não apresenta queixas, com valores de creatinina de 1,8 mg/d e 9,4 mg/d de Hb sérica, decorrente do uso de SRL e ibersatan.

\section{DISCUSSÃO}

Infecção por EBV, infecção gástrica pelo H. pylori e imunodeficiência induzida por medicação imunossupressora constituíram fatores de risco bem conhecidos para desenvolvimento de LGM em nossa paciente. Primeiramente, a infecção por EBV é uma infecção altamente prevalente na população adulta, com aproximadamente $90 \%$ dos indivíduos apresentando evidência de infecção anterior por volta dos 40 anos. Mesmo que o EBV possa ser identificado em muitos linfomas, espera-se que seu impacto no desenvolvimento do LGM seja pequeno, pois a infecção é quase onipresente e a sua prevalência não muda há gerações. Mais ainda, o linfoma gástrico primário raramente tem sido associado ao EBV. ${ }^{3}$ Em segundo lugar, as drogas imunossupressoras após transplante renal aumentam de forma substancial o risco para LGM (30 a 50 vezes). ${ }^{4}$ A estimulação antigênica crônica induzida pelo enxerto e a subsequente imunossupressão provavelmente são os mecanismos determinantes da doença. A proliferação policlonal de células B geralmente tem sido vista primeiro em pacientes transplantados, dos quais $69 \%$ surgem em locais extra-nodais. ${ }^{3} \mathrm{O}$ prognóstico depende da pronta intervenção terapêutica e da redução da imunossupressão. Em terceiro lugar, o fato da infecção por $H$. pylori invariavelmente preceder o desenvolvimento de LGM é agora amplamente aceito. A observação de baixa resposta antigênica do LGM de células B de baixo grau à presença de microorganismo estimulou pesquisadores a testar se a estimulação do tumor mediada por células $\mathrm{T}$ seria interrompida com a erradicação do H. pylori. Publicações recentes confirmaram a regressão do LGM de baixo grau após erradicação do $H$. pylori em até $62 \%$ dos casos num período de 12 meses. ${ }^{5}$ Os LGM de alto grau são geralmente considerados em estágios já independentes da infecção por $H$. pylori, havendo alguns estudos sobre o papel da erradicação do microorganismo nessa situação. Recentemente, têm sido publicados pequenas séries e estudos de casos mostrando que os LGM primários de alto grau podem regredir completamente após alguns meses em até 50\% dos casos após erradicação do microorganismo, especialmente no estágio I. 6

Duas intervenções terapêuticas com intervalo de uma semana foram realizadas para tratar LGM em nossa paciente: tratamento da infecção por H. pylori e modificação da imunossupressão por substituição da CsA e AZA por SRL, com desaparecimento do LGM e persistência da infecção por $H$. pylori nos exames histológicos realizados após um mês de tratamento. Embora o LGM possa desenvolver-se em pacientes de transplante renal e geralmente respondem à erradicação do $H$. pylori, ${ }^{7}$ deve-se considerar que a remissão histológica após a erradicação da bactéria geralmente leva meses ou até um ano, o que não ocorreu com a nossa paciente, que teve regressão rápida do tumor. O SRL, uma lactona macrocíclica produzida pela bactéria streptomyces higroscopicus é uma opção promissora no manuseio de tumores pós-transplante. Inicialmente identificado como um agente fungicida, seu uso tem se estendido como agente imunossupressor para prevenção de rejeição após transplante de órgão sólido e como um agente anti-proliferativo em stents de órgãos para evitar reestenose. Mais recentemente, ele tem sido extensivamente estudado como droga anticancerígena, possuindo ação anti-angiogênese e anti-proliferativa no complexo mTOR. O mTOR é uma quinase serina/treonina que regula o crescimento celular através do complexo-1 do mTOR, cuja ativação tem sido implicada em diversos cânceres humanos. Dados acumulados apóiam o papel do mTOR na linfomagênese e muitos ensaios clínicos estão em andamento com essas drogas, visando o tratamento de diversas malignidades, inclusive do linfoma, sarcoma e glioblastoma. ${ }^{8,9}$

\section{CONCLUSÃO}

No nosso conhecimento este é o primeiro caso de linfoma gástrico MALT em paciente de transplante renal que apresentou pronta e persistente remissão histológica após alteração do esquema imunossupressor com a introdução de SRL. Estas observações devem estimular maiores estudos sobre o papel dessa droga e de outros novos inibidores do mTOR no tratamento de LGM associado ou não à imunossupressão.

\section{ABSTRACT}

B-cell non-Hodgkin lymphoma is the most common post-transplant lymphoproliferative disorder after solid organ transplantation and its treatment is not well defined. We report a case of gastric MALT lymphoma with fast, persistent, and complete remission after conversion of the immunosuppression from cyclosporine (CsA) to sirolimus (SRL). A 42-year-old woman was submitted to renal transplant in 1992 and had a evolution with no major abnormalities until 2006 when a gastroscopy performed to investigate dyspeptic symptoms showed a mixed MALT gastric lymphoma(with low and high grade components) associated with the presence of Helicobacter pylori(H. pylori) infection. 
Two therapeutic interventions in a one-week interval were carried out: treatment of the H. pylori infection (association of omeprazole, amoxicillin and clarithromycin for 14 days) and modification of the immunosuppression by substitution of CsA and azathioprine (AZA) by SRL. Control endoscopy performed one month later showed persistence of $H$. pylori infection and absence of gastric tumor. New endoscopies performed at two-month, seven-month and one year after therapy confirmed the absence of neoplasia and $H$. pylori eradication. Currently, the patient has no complaints, with creatinine values of $1.8 \mathrm{mg} / \mathrm{dl}$ using SRL and ibersatan. SRL has been extensively studied as an anticancer drug, acting as inhibitor of mammalian target of rapamycin (mTOR) inhibitor. Accumulating data support mTOR's role in lymphomagenesis. As far as we know, this is the first report of a gastric MALT lymphoma in a renal transplant patient that presented complete remission after alteration of the immunosuppression scheme with the introduction of SRL.

Keywords: renal transplant, Malt lymphoma, immunosuppression

\section{REFERÊNCIAS}

1. Rabkin CS, Ward MH, Manns A, Blattner WA. Epidemiology of non-Hodgkin's lymphomas. In: Magrath IT, ed. The Non-Hodgkin's Lymphomas. 2nd ed., New York, NY: Oxford University Press. 1997:171-86.

2. Caillard S, Dharnidharka V, Agodoa L, et al: Posttransplant lymphoproliferative disorders after renal transplantation in the United States in era of modern immunosuppression. Transplantation. 2005;80:1233.

3. Wotherspoon AC, Diss TC, Pan L, Singh N, Whelan J, Isaacson PG. Low grade gastric B-cell lymphoma of mucosa associated lymphoid tissue in immunocompromised patients. Histopathology. 1996;28:129-34.

4. Hoover RN. Lymphoma risks in populations with altered immunity-a search for mechanism. Cancer Res. 1992;52 (suppl):5477S-8S.
5. Fischback W, Goebeler-Kolve ME, Dragosics B, Greiner A, Stolte M. Long term outcome of patients with gastric marginal zone B cell lymphoma of mucosa associated lymphoid tissue (MALT) following exclusive Helicobacter pylori eradication therapy. Experience from a large prospective series. Gut. 2004;53:34-7.

6. Sepulveda AR, Coelho LGV. Helicobacter pylori and gastric malignancies. Helicobacter. 2002;7(Suppl. 1):37-42.

7. Ponticelli C, Passerini P. Gastrointestinal complications in renal transplant recipients. Transpl Int. 2005;18:643-50

8. Smith SM. Clinical development of mTOR inhibitors: a focus on lymphoma. Rev Recent Clin Trials. 2007;2:103-10.

9. Easton JB, Houghton PJ. mTOR and cancer therapy. Oncogene. 2006;16;25:6436-46. 